\title{
Neurological maturation of late preterm infants at 34 wk assessed by amplitude integrated electroencephalogram
}

\author{
Ross Sommers ${ }^{1}$, Richard Tucker ${ }^{1}$, Chellamani Harini ${ }^{2}$ and Abbot R. Laptook ${ }^{1}$
}

BACKGROUND: This study tested if measures of central nervous system (CNS) immaturity reflected by amplitude integrated electroencephalogram (aEEG) and associated clinical morbidities are determinants of length of hospitalization among late preterm infants born at $34 \mathrm{wk}$.

METHODS: This was a prospective cohort study of infants with a gestational age of $34 \mathrm{wk}$ 0-6 d who had a single aEEG recording acquired over $6 \mathrm{~h}$ in a neonatal intensive care unit within $72 \mathrm{~h}$ of birth $(n=80)$. Infants were followed for predefined morbidities (classified as CNS or non-CNS) and length of hospitalization (determined by the clinical care team). aEEG variables were correlated with length of hospitalization.

RESULTS: Eighty infants were enrolled and 75 aEEG recordings were analyzed. The average length of hospitalization was $10.4 \pm 7.2 \mathrm{~d}$ (range 3-46 d). The total number of cycles recorded in the first $72 \mathrm{~h}$ following birth were inversely correlated with the length of hospitalization $\left(r^{2}=0.44, P<0.001\right)$. Kaplan-Meier curves indicated that morbidities consistent with neurological immaturity were associated with a longer length of hospitalization $(P<0.001)$.

CONCLUSION: Neurological maturation as indicated by aEEG and specific clinical morbidities is an important determinant of length of hospitalization among late-preterm infants.

$\mathbf{L}^{2}$ ate preterm infants (gestational age of 34 wk 0-6 d) rep- $>70 \%$ of all infants born $<37$-wk gestation (1) Late preterm infants have increased morbidities and require disproportionate healthcare resources compared with term infants $(2,3)$. Short term morbidities encountered in late preterm infants include respiratory distress syndrome (RDS), retained lung fluid, sepsis evaluations, temperature instability, hyperbilirubinemia, hypoglycemia, apnea, and poor feeding (4-9). These morbidities reflect incomplete maturation of multiple organ systems including the central nervous system (CNS) (10). At $34 \mathrm{wk}$, the brain weighs only $65 \%$ of the term brain weight and an increase in synaptogenesis and dendritic pruning accompanies further growth until term (10). These observations suggest that CNS-related morbidities may be prominent among late preterm infants at $34 \mathrm{wk}$ and contribute to the length of hospitalization. Understanding the ontogeny of brain growth, the frequency of apnea, and maturation of breathing and deglutition among late preterm infants has been identified as important research areas (1).

The amplitude-integrated electroencephalogram (aEEG) is a bedside device to monitor cerebral electrical activity. It provides an index of cerebral background activity and correlates with the background pattern of a full EEG (11). Premature infants undergoing serial aEEG recordings after birth demonstrate maturational changes characterized by increasing frequency of cycles (derived from repetitive changes between continuous and discontinuous EEG activity), decreasing discontinuity and a narrowing of the span (12). These changes suggest that aEEG maybe a potential neurophysiologic marker for CNS-related morbidities among late preterm infants. We postulated that the extent of neurological maturation of infants born at $34 \mathrm{wk}$ is an important determinant of the length of hospitalization. To test this hypothesis, we acquired aEEG recordings in the first $72 \mathrm{~h}$ following birth to determine if a more immature pattern was associated with a longer length of hospitalization. A secondary-related hypothesis was that longer hospitalizations for infants born at $34 \mathrm{wk}$ are more frequently associated with morbidities attributable to CNS immaturity compared with non-CNS morbidities.

\section{RESULTS}

During the study interval, 234 infants were admitted to the neonatal intensive care unit (NICU) between $34 \mathrm{wk} 0-6 \mathrm{~d} ; 92$ mothers were not approached (dating by last menstrual period only), 18 refused consent, and 44 were not approached (investigator unavailable), leaving enrollment of 80 infants. Maternal and infant characteristics are listed in Table 1 . None of the infants had fetal acidemia $(\mathrm{pH}<7.0)$ at or after birth. Length of hospitalization ranged from 3 to $46 \mathrm{~d}$ with an average of $10.4 \pm 7.2 \mathrm{~d}$ (median, $9 \mathrm{~d}$, interquartile range, 6-12 d).

Five infants had aEEG recordings that could not be evaluated ( 2 - $h$ recording in two, poor impedance in three). The age at aEEG acquisition was $26 \pm 15 \mathrm{~h}$ and the cycle frequency or length of hospitalization did not differ with postnatal age at aEEG (Table 2). There was wide variability in aEEG findings with immature patterns (lower border (LB) near 5 microvolts, few cycles) and more mature patterns (elevated LB, well defined cycles) (Figure 1). The distribution of cycle type was 
Table 1. Maternal and infant characteristics

\begin{tabular}{lc} 
Maternal & \\
Age & $27 \pm 7 \mathrm{y}$ \\
Gestational diabetes & $8 \%$ \\
Twins & $11 \%$ \\
Pre-eclampsia & $17 \%$ \\
Placental abruption & $5 \%$ \\
Premature rupture of membranes & $4 \%$ \\
Preterm labor & $43 \%$ \\
Betamethasone & $16 \%$ \\
Cesarean section & $41 \%$ \\
Infant & \\
Gestational age (wk, d) & $34 \mathrm{wk} 3 \mathrm{~d} \pm 2 \mathrm{~d}^{\mathrm{b}}$ \\
Birth weight (g) & $2,338 \pm 330 \mathrm{~g}^{\mathrm{b}}$ \\
Male & $58 \%$ \\
White/black/Hispanic/other & $50 / 12 / 12 / 26 \%$ \\
Apgar-1 min & $8(8,9)^{\mathrm{c}}$ \\
Apgar-5 min & $9(8,9)^{\mathrm{c}}$ \\
\hline
\end{tabular}

aReceipt within 2 wk of delivery. ${ }^{b}$ Mean \pm SD. ${ }^{c}$ Median (interquartile range).

Table 2. Mean number of cycles per hour and length of hospitalization as a function of age at acquisition

\begin{tabular}{lccc}
\hline Age of aEEG acquisition & $n$ & $\begin{array}{c}\text { Cycles } \\
\text { (number/h) }\end{array}$ & $\begin{array}{c}\text { Length of } \\
\text { hospitalization (d) }\end{array}$ \\
\hline From birth to 12 h & 18 & $0.55 \pm 0.14$ & $11.7 \pm 9.6$ \\
$>12-24 \mathrm{~h}$ & 21 & $0.58 \pm 0.13$ & $11.8 \pm 9.3$ \\
$>24-36 \mathrm{~h}$ & 19 & $0.55 \pm 0.09$ & $11.5 \pm 5.9$ \\
$>36-48 \mathrm{~h}$ & 7 & $0.67 \pm 0.14$ & $7.2 \pm 2.1$ \\
$>48 \mathrm{~h}$ & 10 & $0.59 \pm 0.14$ & $10.3 \pm 4.7$ \\
\hline
\end{tabular}

aEEG, amplitude integrated electroencephalogram.

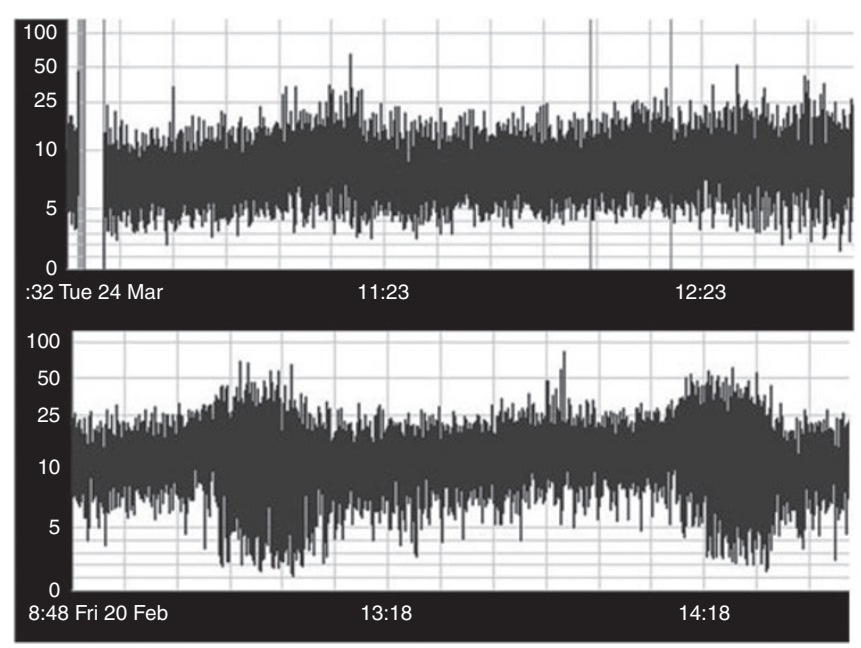

Figure 1. Amplitude integrated electroencephalogram recordings from two infants. The top panel shows an immature pattern: lower border predominately at or below 5 micro-volts and without cycles $>2.5 \mathrm{~h} \mathrm{(23-d}$ hospital stay). The lower panel shows a mature pattern: elevated lower border during noncycle activity and two cycles (3-d hospital stay).
43,47 , and $10 \%$ for mature, immature, and interrupted, respectively. There was moderately good interobserver reliability for classifying mature, immature, and interrupted cycles (kappa, $0.77,0.70$, and 0.67 , respectively).

There was a total of $0.57 \pm 0.13$ cycles/h (range $0.25-0.73$ ). An increasing frequency of cycles/h correlated with a shorter hospitalization and a polynomial regression accounted for more of the variance compared with a linear regression (polynomial: $r^{2}=0.44, P<0.01$, linear: $r^{2}=0.34, P<0.01$, Figure 2a). Similarly, an increasing frequency of cycles/h correlated with a shorter length of hospitalization for infants with RDS, and for infants without RDS (Figure 2b). However the relationship differed among infants with RDS since the hospitalization was longer ( $14.6 \pm 10.2$ vs. $10.0 \pm 6.4 \mathrm{~d}$, RDS vs. no RDS, respectively, $P=0.028)$ but the frequency of cycles/h was similar $(.56 \pm 0.14$ vs. $0.58 \pm 0.13$ cycles/h with and without RDS, respectively).

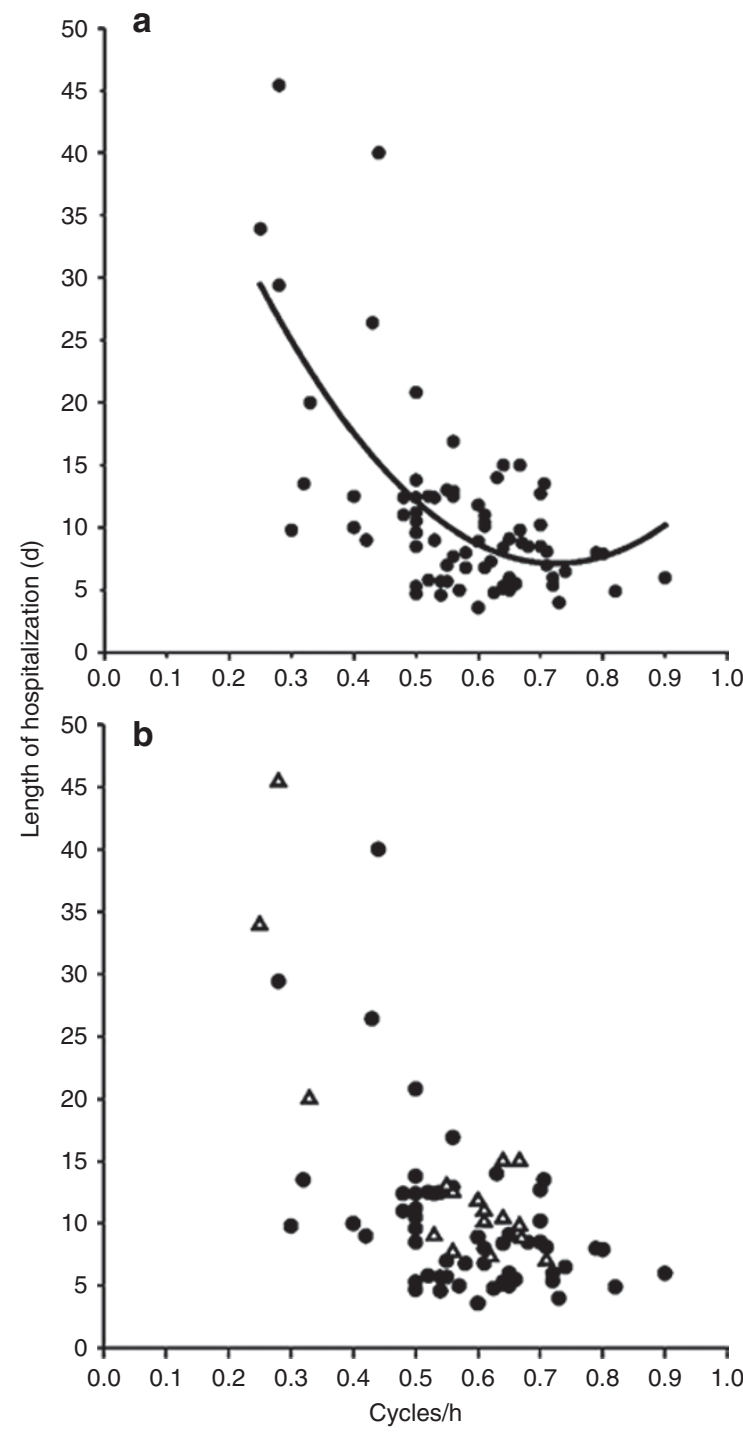

Figure 2. The relationship between total cycles/h and hospital stay. (a) Increasing cycle number was associated with decreasing hospital stay $\left(y=59.1-143.5 x+99 x^{2}, r^{2}=0.44, P<0.001\right)$ for all infants. Panel $\mathbf{b}$ displays the same cohort of infants but indicates the presence or absence of RDS by open triangles and closed circles, respectively. 
For infants with RDS the quadratic regression (hospitalization duration $=89.3-2.54$ cycles +2.04 cycles $^{2}$ ) was improved by the cycle ${ }^{2}$ term $(P=0.017$.) but not for infants without RDS (hospitalization duration $=41.6-0.97$ cycles +0.97 cycles $\left.^{2}\right)(P$ $=0.10)$. Increasing ratios of mature to total cycles correlated with a shorter hospitalization $\left(r^{2}=0.07, P=0.02\right)$ and increasing ratios of immature plus interrupted to total cycles correlated with a longer hospitalization $\left(r^{2}=0.08, P=0.02\right)$. An aEEG with $<0.56$ cycles/h predicted a prolonged hospitalization with a sensitivity of $87 \%$, specificity of $57 \%$, positive predictive value of $20 \%$, and a negative predictive value of $97 \%$.

The average LB and span voltages for intercycle intervals were $7.6 \pm 1.1 \mathrm{mcv}$ and $10.2 \pm 2.2 \mathrm{mcv}$, respectively. A longer hospitalization was associated with decreasing LB $\left(r^{2}=\right.$ $0.1, P<0.01)$ but not the span. The LB of infants with RDS $(7.2 \pm 1.3 \mathrm{mcv})$ was marginally lower than infants without RDS $(7.7 \pm 0.91 \mathrm{mcv}, P=0.049)$ but there were no differences in span. Fifty-six and 24 infants were studied in the old and new NICU, respectively, and the cycle frequency and length of hospitalization was similar. There were no differences in aEEG variables by gender or mode of delivery.

Table 3. The incidence and duration of neonatal morbidities

\begin{tabular}{|c|c|c|c|c|}
\hline \multirow[b]{2}{*}{ CNS morbidity } & \multirow[b]{2}{*}{$\begin{array}{c}\text { Incidence } \\
\%\end{array}$} & \multicolumn{3}{|c|}{ Duration } \\
\hline & & $\begin{array}{l}\text { Median } \\
\text { (d) }\end{array}$ & 25 th-75th & Range \\
\hline $\begin{array}{l}\text { Immature suck } \\
\text { swallow }\end{array}$ & 76 & 6 & $4-9$ & $3-46$ \\
\hline Desaturations & 50 & 6 & $4-8$ & $3-41$ \\
\hline Apneas & 29 & 3 & $2-6$ & $2-25$ \\
\hline \multicolumn{5}{|l|}{ Non-CNS morbidity } \\
\hline $\begin{array}{l}\text { Respiratory distress } \\
\text { syndrome }\end{array}$ & 23 & 4 & $3-5$ & $2-18$ \\
\hline Pneumonia & 2.5 & 5.5 & $5.3-5.8$ & $5-6$ \\
\hline $\begin{array}{l}\text { Transient tachypnea } \\
\text { of the newborn }\end{array}$ & 10 & 1.5 & $1-2$ & $1-3$ \\
\hline $\begin{array}{l}\text { Temperature } \\
\text { instability }\end{array}$ & 79 & 5 & $3-6$ & $2-18$ \\
\hline $\begin{array}{l}\text { Gastrointestinal } \\
\text { intolerance }\end{array}$ & 55 & 5 & $4-7$ & $1-13$ \\
\hline Hyperbilirubinemia & 40 & 5 & $4-6$ & $2-13$ \\
\hline Sepsis evaluation & 62 & 2 & $2-2$ & $2-7$ \\
\hline Hypoglycemia & 6 & 1 & $1-1.5$ & $1-3$ \\
\hline $\begin{array}{l}\text { Necrotizing } \\
\text { enterocolitis }\end{array}$ & 1 & 7 & & 7 \\
\hline \multicolumn{5}{|c|}{$\begin{array}{l}\text { Immature suck swallow coordination was inferred from feedings interrupted by } \\
\text { choking events or slow feeding requiring intravenous supplementation or use of a } \\
\text { gavage tube. Desaturations were falls in oxygen saturations }<80 \% \text { with or without } \\
\text { feeding. Apnea were pauses in respirations }>20 \text { s. Respiratory morbidities (RDS, TTN, } \\
\text { pneumonia) were distinguished by their course and characteristic radiographic } \\
\text { findings. Temperature instability was defined by the use of exogenous heat to maintain } \\
\text { body temperature. Gastrointestinal intolerance was defined by episodes of emesis, } \\
\text { residuals, or abdominal distension. Hyperbilirubinemia was defined by elevated } \\
\text { bilirubin concentrations requiring phototherapy. Sepsis evaluations were determined } \\
\text { by the use of antibiotics. Hypoglycemia was determined by a blood glucose } \\
\text { concentration }<40 \text { mg\%. Necrotizing enterocolitis was defined by Bell's criteria. }\end{array}$} \\
\hline $\begin{array}{l}\text { CNS, central nervous syste } \\
\text { tachypnea of the newbor }\end{array}$ & RDS, respirato & distress syno & me;TTN, transi & \\
\hline
\end{tabular}

CNS and non-CNS morbidities were common (Table 3). Eleven (12\%) infants required ventilator support (median duration, $1.5 \mathrm{~d}$ ) and 8 (10\%) received surfactant. Twenty (25\%) infants received continuous positive airway pressure (median duration, $2 \mathrm{~d}$ ) and $17(21 \%)$ received nasal cannula (median duration, $3 \mathrm{~d}$ ). There were no deaths, positive blood cultures, caffeine use, volume expansion, pressor support and one infant was discharged home on an apnea monitor.

The average length of a short and prolonged hospitalization (1st and 4th quartile, respectively) was $4.8 \pm 1$ and $19.4 \pm 9.7 \mathrm{~d}$, respectively. Morbidities persisting closest to the time of discharge were CNS related for 18 of the 20 infants with a prolonged hospitalization (desaturation events in 11, immature suck-swallow in 7) compared with only 5 of the 20 infants with a short hospitalization $\left(P<0.01, \chi^{2}\right)$. Kaplan-Meier curves demonstrated that duration of hospitalization was longer for those with prolonged CNS-related morbidities compared with non-CNS morbidities $(P<0.001$, Figure 3$)$.

\section{DISCUSSION}

The results of this investigation support a heterogeneous neurological maturation among infants born at $34 \mathrm{wk}$, which is an important determinant of the length of hospitalization. The aEEG acquired in the first $72 \mathrm{~h}$ after birth indicated an inverse relationship between the number of cycles/h and length of hospitalization; specifically, fewer cycles/h, as observed with greater immaturity, was associated with a longer length of hospitalization. The relationship was present irrespective of the presence or absence of RDS, but was more pronounced for infants with RDS. Parallel to these findings was that CNSrelated morbidities were more commonly associated with a longer hospitalization compared with non-CNS morbidities.

Enrollment was purposefully limited to 34-wk gestation. This limits gestational age related changes in aEEG (12-14) and focuses on the lower gestational age of late preterm infants who are known to have a higher frequency of morbidities which may require intensive care compared with infants at

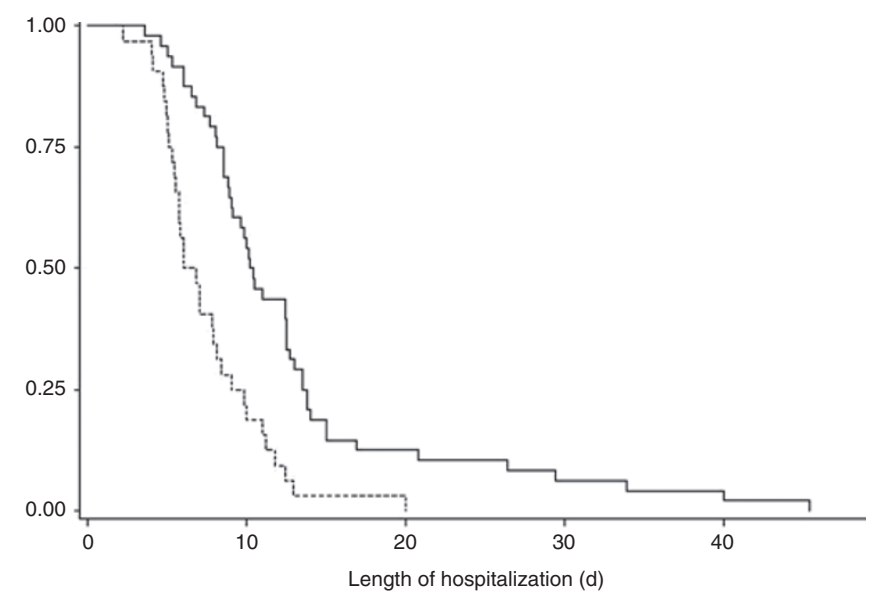

Figure 3. Kaplan-Meier curves comparing the duration of hospitalization for any CNS-related and any non-CNS morbidity. Infants with CNS morbidities are indicated by the solid line and infants without CNS morbidities are indicated by the dashed line. CNS, central nervous system. 


\section{Articles $\mid$ sommers et al.}

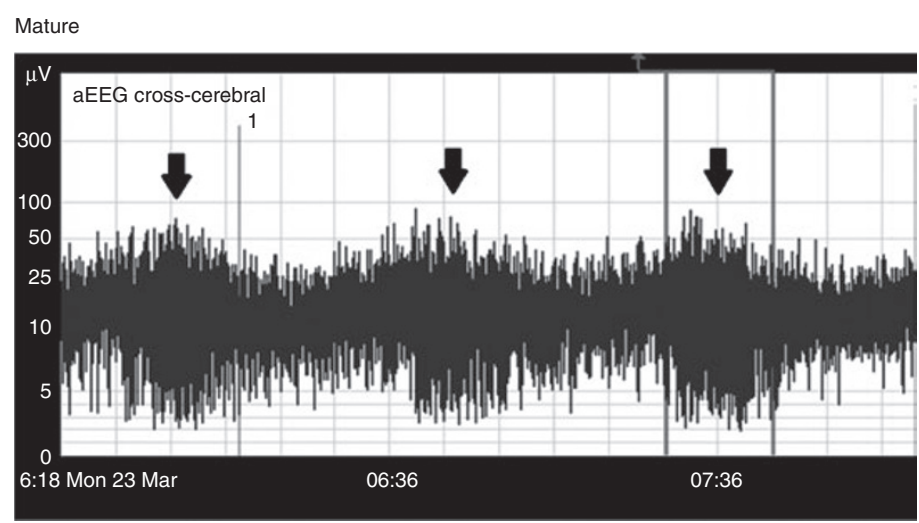

Immature
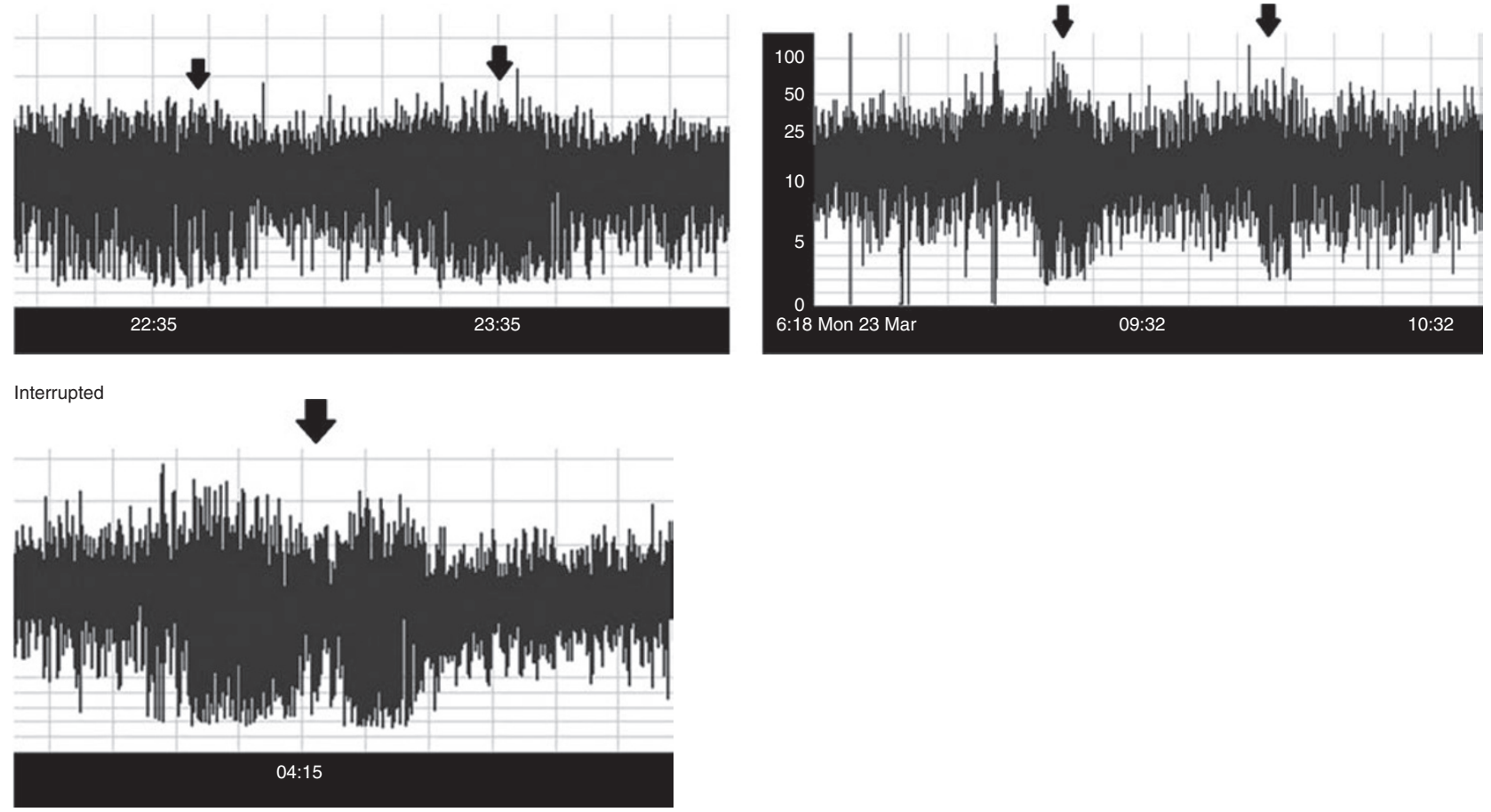

Figure 4. Examples of different cycles (labeled with an arrow) with time on the $x$-axis and amplitude integrated electroencephalogram voltage (microvolts) on the $y$-axis. The top tracing represents a mature cycle. The middle two tracings represent immature patterns. The bottom tracing represents an interrupted pattern with two cycle intervals separated by noncycle activity.

$35-36$ wk $(9,15-17)$. Thus, accurate gestational age was criti$\mathrm{cal}$ and infants were enrolled only if a first trimester sonogram was available (18). Exclusion criteria avoided conditions which may alter the aEEG recording independent of maturation.

In this investigation, intermittent widening of the aEEG span was classified as a cycle and was not labeled a sleep-wake cycle or quiet sleep (19). We did not combine the aEEG with observations of movement, behavior or rapid eye movements to characterize sleep, wakefulness or transitions between these states. Although sleep-wake states can be identified in preterm infants (20), cohorts studied are usually stable without morbidities of prematurity $(21,22)$. We examined the aEEG and specifically the number of cycles as a neurophysiologic marker to determine if it could indicate neurological maturation for infants of a single week of gestation. A low frequency of cycles was not considered a manifestation of EEG suppression following brain injury (e.g., hypoxia-ischemia) since none of the infants had supportive clinical data (fetal acidemia, low Apgars, need for resuscitation) or exposure to drugs known to suppress EEG activity. Cycles have been demonstrated in infants studied between 25 and $30 \mathrm{wk}$ by the presence of alternating intervals of EEG discontinuity (23). The presence of cycles on aEEG has been reported as early as $26 \mathrm{wk}(24,25)$. However, determination of cycles is difficult in extremely preterm neonates, since much of the aEEG tracing is discontinuous. These observations may explain the only moderate extent of agreement for determination of cycles among two observers examining the aEEG for this study. These considerations have led to tier classification of cycle appearance, including mature, imminent or immature, interrupted and absence of cycles $(13,26)$. Our results indicate that many infants at 34 -wk gestation have cycles with an immature or interrupted appearance. 
The evolution of cycles with increasing postnatal age remains uncertain without subsequent recordings.

A lower number of cycles/h in the first 72 postnatal $h$ was associated with a longer hospitalization. Similarly, persistence of CNS clinical morbidities was associated with a longer hospitalization. Our results demonstrate a high frequency, prolonged duration, and overlap in time of apparent immature suck-swallow coordination, desaturation events and apnea that are consistent with the ongoing maturation of feeding and respiratory events in the late third trimester $(27,28)$. These observations support the neurological state at birth as an important determinant of length of hospitalization among infants at 34-wk gestation. Whether there are important differences in the association between cycles and length of hospitalization among infants with and without RDS is unclear given the small cohort with RDS. In spite of the association, the low specificity and positive predictive value between cycles and length of hospitalization, as well as only moderate level of agreement for determination of cycles indicates that aEEG is not helpful as a screening tool or in the clinical decisions regarding specific late preterm infants. The LB of the aEEG was also inversely correlated with length of hospitalization but the LB accounted for less of the variance in hospital stay than total cycles.

The aEEG recordings were acquired within $72 \mathrm{~h}$ of birth and some at $<24 \mathrm{~h}$ of age. There has been concern that the birth process may disrupt infant state and prevent reliable characterization of state immediate postnatally (29). Whether this pertains to aEEG is unclear since we did not perform serial measurements among study subjects. However, there were no differences in cycles/h when day of aEEG acquisition was compared. Furthermore, the extra-uterine environment is associated with maturation of the aEEG $(30,31)$. Therefore, we balanced aEEG acquisition that would represent maturation at birth and allow a feasible approach for recruitment.

There are limitations to this study. Enrollment was limited to $34 \mathrm{wk}$ and results may have been more robust if all late preterm infants were studied. Unfortunately, this was not feasible since many late preterm infants especially at 35- and 36-wk gestational ages were well enough for care outside of a NICU. This investigation did not have a large enough sample size to rigorously examine other variables that may affect organ maturation such as maternal diabetes and gender $(32,33)$. We used length of hospitalization as a proxy for resolution of clinical morbidities. It is acknowledged that wide variations in the length of stay for premature infants may also reflect management of specific morbidities (e.g., apnea) (34-36) and even reflect unit census (37). Since our observations were from a single NICU, physician or clinical team preference may influence recognition of mature behavior and affect length of hospitalization. Nonetheless, the time of discharge was determined by the clinical care team reflecting 14 different Attending Physicians, and the characterization of clinical morbidities including CNS- or non-CNS-related grouping was done prospectively. Finally, the aEEG recording represents only one time point and serial aEEG recordings may provide important information.
The predictive indices for the association between total number of cycles and length of hospitalization do not support use of aEEG as a helpful tool for the clinical management of late preterm infants or counseling their families. Furthermore, transient aEEG suppression secondary to brain injury or specific drugs are important caveats in the interpretation of aEEG. In the absence of such conditions, the associations between both aEEG cycles and CNS clinical morbidities with length of hospitalization add to our understanding of late preterm infants. These observations provide valuable insights into a neurophysiologic basis for a substantial portion of the variance in duration of hospitalization for this cohort of late preterm infants.

\section{METHODS}

\section{Study Design}

This is a prospective cohort study of infants admitted to the NICU of Women \& Infants Hospital of Rhode Island and was performed from September 2008 to April 2010 after Institutional Review Board approval and parental consent.

\section{Inclusion and Exclusion Criteria}

Entry criteria included a gestational age of 34wk 0-6 d using first trimester ultrasound dating and NICU admission. During the study, all infants of 34-wk gestation were triaged to the NICU for a $6 \mathrm{~h}$ observation period; infants could room in with the mother or go to the Newborn Nursery if transition was successful. However, most Attending Physicians used $<35 \mathrm{wk}$ as an admission criterion due to morbidities remote from birth. Exclusion criteria included small for gestational age $(<1600 \mathrm{~g})$, major anomalies, clinical seizures, maternal substance abuse, and congenital infections.

\section{Study Intervention}

Infants had a single 6-h aEEG recording at the bedside in the first $72 \mathrm{~h}$ of life and were prospectively followed for predefined morbidities and length of hospitalization. From September 2008 until September 2009 , infants were cared for in open bays and after which infants were cared for in single patient/family rooms of a new NICU.

\section{Amplitude Integrated EEG Acquisition and Analysis}

All recordings were performed using the BrainZ BRM3 monitor (Natus Medical, San Carlos CA) using five disposable hydrogel electrodes in the C3-P3 and C4-P4 locations of the head and a ground lead on the shoulder. The skin was prepared using Nu-Prep and impedance was continuously monitored. aEEG voltage values were determined by the BRM3 and displayed by the BrainZ Analyze offline software. Only the cross-cerebral (P3-P4) channel was analyzed.

Each aEEG was analyzed independently by two investigators (RS and $\mathrm{CH}$ ) without knowledge of the length of hospitalization. The total number of cycles was determined and represented the primary predictor variable. The term cycles was used in preference to sleep-wake cycles since data on the behavioral state was not recorded. Cycles represent the repetitive changes between continuous and discontinuous EEG activity as described by Kidokoro et al. (19). Cycle type was prospectively defined based on published criteria (12-14) and are described below (Figure 4). Cycles were visually identified and Analyze was used in cases of uncertainty to establish cycle criteria. Differences were adjudicated between investigators.

Mature Cycle: Criteria included all of the following: (i) duration of 20-60 min, (ii) upper border voltage increase by at least $10 \%$ compared with the preceding or subsequent $20 \mathrm{~min}$ of aEEG, and (iii) LB voltage decrease to either at least $5 \mathrm{mcv}$ for $50 \%$ of the cycle duration, or at least $15 \%$ lower than 20 minutes of the preceding or subsequent aEEG.

Immature Cycle: Criteria included either (i) duration of 20-60 minutes with a LB voltage as for mature cycles or (ii) duration of 10-20 minutes with an upper and LB voltage as for mature cycles.

Interrupted Cycle: Criteria included an interval that fulfills the mature cycle criteria but is interrupted by $<20$ minutes of noncycle 
activity. This gives the appearance of two individual cycles and a total duration $<60$ minutes.

After identifying cycles, aEEG data were exported as a Windows Excel file to display upper and LB voltages at 1 minute intervals. Voltage values were then calculated for the cycle and noncycle intervals. Variables included LB voltage, span (upper and LB voltage difference), and discontinuity (the time voltage activity $<5 \mathrm{mcv}$ ). Frequency of cycles was calculated from the number of cycles and the recording length.

\section{Neonatal Morbidities}

The medical records of mother and infant were reviewed. Neonatal morbidities were prospectively defined, assigned by one author (R.S.) and included RDS, transient tachypnea, pneumonia, temperature instability, sepsis evaluations, hyperbilirubinemia, feeding intolerance, hypoglycemia, necrotizing enterocolitis, immature suck-swallow coordination, desaturation episodes, and apnea (definitions listed with Table 3). Duration of morbidities was determined when specific therapy was discontinued (e.g., exogenous heat for temperature instability, phototherapy for hyperbilirubinemia, antibiotics for sepsis or necrotizing enterocolitis) or when symptoms resolved (RDS, transient tachypnea, pneumonia, apnea or desaturation events $<80 \%$ with or without feeds). The duration of feeding intolerance was the time to tolerate feeds without emesis, gastric residuals or abdominal distension. The duration of immature suck swallow coordination was inferred from the time until all feeds (breast or bottle) were taken without intravenous supplementation or gavage tube in $<30$ minutes per feed for all feeds within a calendar day. Morbidities were grouped prospectively as a CNS origin (immature suck swallow coordination, desaturation episodes, and apnea) or as non-CNS (all other morbidities).

Gestational dating was determined by ultrasound within the first 12 wk of pregnancy. Data was collected for maternal and obstetric variables, infant demographics and delivery room events. Length of hospitalization in days was calculated by dividing the amount of hours after admission to the NICU from the electronic chart by 24 . Discharge criteria included resolution of all morbidities, maintenance of body temperature in a bassinet for at least $24 \mathrm{~h}$, breathing room air, and ability to consume each feed by breast or bottle within thirty minutes over a 24 -h interval. Infants were maintained on cardio-respiratory monitors and pulse oximeters to the time of discharge. The discharge date was determined by the attending physician responsible for the clinical service.

\section{Sample Size and Data Analysis}

Data regarding specific aEEG variables among infants at $34 \mathrm{wk}$ is limited and therefore sample size was based on differences in length of hospitalization. Over 3 months preceding this study, infants born at 34 wk had a median hospitalization of $11 \mathrm{~d}$ (range 2-34 d) with the 25th and 75th\% (short and prolonged hospitalization) of 7 and $13 \mathrm{~d}$, respectively. Using a two-sided $t$-test, an alpha at 0.05 and power of $80 \%, 80$ infants are required to detect a mean difference of $6 \mathrm{~d}$ and was used as our effect size given the absence of aEEG data at 34-wk gestational age.

Demographic and clinical variables were compared by $t$-test or analysis of variance for continuous variables and the $\chi^{2}$ test for categorical variables. Interobserver cycle classification was compared by kappa scores. The relationship between aEEG variables and length of hospitalization was estimated by linear and polynomial regression analysis. The length of hospitalization was rank ordered, divided into quartiles and the first and fourth quartiles were deemed a short or prolong hospitalization, respectively. The association between cycles and length of hospitalization was subjected to receiver operator curve analysis to determine predictive indices of a prolonged hospitalization. Kaplan-Meier curves of morbidity that persisted closest to time of discharge (any CNS or non-CNS) were compared with the log rank test. Data are represented as mean \pm SD or median with interquartile ranges.

\section{ACKNOWLEDGMENTS}

Ross Sommers did the literature search to define the gap in knowledge, helped with design and writing of the protocol, recruited all patients, per- formed the aEEG recordings, analyzed the data and wrote the drafts of the paper. Richard Tucker provided statistical support for the paper, including sample size determination and data analysis (Kaplan-Meier curves, log rank test, linear and polynomial regressions). He reviewed the paper for use of the statistics. Chellamani Harini participated in the interpretation of aEEG and helped with the analysis of the aEEG data. She reviewed the drafts of the paper. Abbot Laptook provided mentorship for Ross Sommers and was involved in all aspects of the study, including concept development, writing the protocol, data interpretation, and review of all drafts.

\section{REFERENCES}

1. Raju TN, Higgins RD, Stark AR, Leveno KJ. Optimizing care and outcome for late-preterm (near-term) infants: a summary of the workshop sponsored by the National Institute of Child Health and Human Development. Pediatrics 2006;118:1207-14.

2. McIntire DD, Leveno KJ. Neonatal mortality and morbidity rates in late preterm births compared with births at term. Obstet Gynecol 2008;111:3541.

3. Bird TM, Bronstein JM, Hall RW, Lowery CL, Nugent R, Mays GP. Late preterm infants: birth outcomes and health care utilization in the first year. Pediatrics 2010;126:e311-9.

4. Adamkin DH. Feeding problems in the late preterm infant. Clin Perinatol 2006;33:831-7; abstract ix.

5. Adamkin DH. Late preterm infants: severe hyperbilirubinemia and postnatal glucose homeostasis. J Perinatol 2009;29:Suppl 2:S12-7.

6. Engle WA, Tomashek KM, Wallman C; Committee on Fetus and Newborn, American Academy of Pediatrics. "Late-preterm" infants: a population at risk. Pediatrics 2007;120:1390-401.

7. Hibbard JU, Wilkins I, Sun L, et al. Respiratory morbidity in late preterm births. JAMA 2010;304:419-25.

8. Raju TN. Late-preterm births: challenges and opportunities. Pediatrics 2008;121:402-3.

9. Wang ML, Dorer DJ, Fleming MP, Catlin EA. Clinical outcomes of nearterm infants. Pediatrics 2004;114:372-6.

10. Kinney HC. The near-term (late preterm) human brain and risk for periventricular leukomalacia: a review. Semin Perinatol 2006;30:81-8.

11. Toet MC, van der Meij W, de Vries LS, Uiterwaal CS, van Huffelen KC. Comparison between simultaneously recorded amplitude integrated electroencephalogram (cerebral function monitor) and standard electroencephalogram in neonates. Pediatrics 2002;109:772-9.

12. Kuint J, Turgeman A, Torjman A, Maayan-Metzger A. Characteristics of amplitude-integrated electroencephalogram in premature infants. J Child Neurol 2007;22:277-81.

13. Burdjalov VF, Baumgart S, Spitzer AR. Cerebral function monitoring: a new scoring system for the evaluation of brain maturation in neonates. Pediatrics 2003;112:855-61.

14. Thornberg E, Thiringer K. Normal pattern of the cerebral function monitor trace in term and preterm neonates. Acta Paediatr Scand 1990;79:20-5

15. Teune MJ, Bakhuizen S, Gyamfi Bannerman C, et al. A systematic review of severe morbidity in infants born late preterm. Am J Obstet Gynecol 2011;205:374.e1-9.

16. Engle WA. Morbidity and mortality in late preterm and early term newborns: a continuum. Clin Perinatol 2011;38:493-516.

17. Dimitriou G, Fouzas S, Georgakis V, et al. Determinants of morbidity in late preterm infants. Early Hum Dev 2010;86:587-91.

18. Gardosi J, Geirsson RT. Routine ultrasound is the method of choice for dating pregnancy. Br J Obstet Gynaecol 1998;105:933-6.

19. Kidokoro $\mathrm{H}$, Inder $\mathrm{T}$, Okumura $\mathrm{A}$, Watanabe $\mathrm{K}$. What does cyclicity on amplitude-integrated EEG mean? J Perinatol 2012;32:565-9.

20. Greisen G, Hellström-Vestas L, Lou H, Rosen I, Svenningsen N. Sleepwaking shifts and cerebral blood flow in stable preterm infants. Pediatr Res 1985;19:1156-9.

21. Ardura J, Andrés J, Aldana J, Revilla MA. Development of sleep-wakefulness rhythm in premature babies. Acta Paediatr 1995;84:484-9.

22. Ingersoll EW, Thoman EB. Sleep/wake states of preterm infants: stability, developmental change, diurnal variation, and relation with caregiving activity. Child Dev 1999;70:1-10. 
23. Scher MS, Johnson MW, Holditch-Davis D. Cyclicity of neonatal sleep behaviors at 25 to 30 weeks' postconceptional age. Pediatr Res 2005;57:87982.

24. Hellström-Westas L, Rosén I, Svenningsen NW. Cerebral function monitoring during the first week of life in extremely small low birthweight (ESLBW) infants. Neuropediatrics 1991;22:27-32.

25. Olischar M, Klebermass K, Kuhle S, et al. Reference values for amplitudeintegrated electroencephalographic activity in preterm infants younger than 30 weeks' gestational age. Pediatrics 2004;113(1 Pt 1):e61-6.

26. Hellstrom-Westas L. Amplitude-integrated EEG classification and interpretation in preterm and term infants. NeoReviews 2006;7:e76-87.

27. Darnall RA, Ariagno RL, Kinney HC. The late preterm infant and the control of breathing, sleep, and brainstem development: a review. Clin Perinatol 2006;33:883-914; abstract x.

28. Barlow SM. Oral and respiratory control for preterm feeding. Curr Opin Otolaryngol Head Neck Surg 2009;17:179-86.

29. Carroll DA, Denenberg VH, Thoman EB. A comparative study of quiet sleep, active sleep, and waking on the first 2 days of life. Dev Psychobiol 1999;35:43-8.

30. Soubasi V, Mitsakis K, Nakas CT, et al. The influence of extrauterine life on the aEEG maturation in normal preterm infants. Early Hum Dev 2009;85:761-5.
31. Klebermass K, Kuhle S, Olischar M, Rücklinger E, Pollak A, Weninger M. Intra- and extrauterine maturation of amplitude-integrated electroencephalographic activity in preterm infants younger than 30 weeks of gestation. Biol Neonate 2006;89:120-5.

32. Bourbon JR, Farrell PM. Fetal lung development in the diabetic pregnancy. Pediatr Res 1985;19:253-67.

33. Hanley K, Rassner U, Jiang Y, et al. Hormonal basis for the gender difference in epidermal barrier formation in the fetal rat. Acceleration by estrogen and delay by testosterone. J Clin Invest 1996;97:2576-84 .

34. Altman M, Vanpée M, Cnattingius S, Norman M. Moderately preterm infants and determinants of length of hospital stay. Arch Dis Child Fetal Neonatal Ed 2009;94:F414-8.

35. Eichenwald EC, Blackwell M, Lloyd JS, Tran T, Wilker RE, Richardson DK. Inter-neonatal intensive care unit variation in discharge timing: influence of apnea and feeding management. Pediatrics 2001;108:928-33.

36. Profit J, Zupancic JA, McCormick MC, et al. Moderately premature infants at Kaiser Permanente Medical Care Program in California are discharged home earlier than their peers in Massachusetts and the United Kingdom. Arch Dis Child Fetal Neonatal Ed 2006;91:F245-50.

37. Profit J, McCormick MC, Escobar GJ, et al. Neonatal intensive care unit census influences discharge of moderately preterm infants. Pediatrics 2007;119:314-9. 